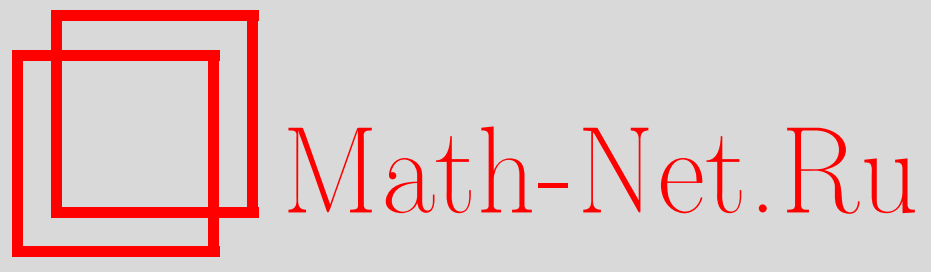

В. Ю. Протасов, О числе замкнутых геодезических на многограннике, УМН, 2008, том 63, выпуск 5, 197198

DOI: https://doi.org/10.4213/rm9228

Использование Общероссийского математического портала Math-Net.Ru подразумевает, что вы прочитали и согласны с пользовательским соглашением http://www .mathnet.ru/rus/agreement

Параметры загрузки:

IP : 35.174 .16 .151

26 апреля 2023 г., 15:26:47

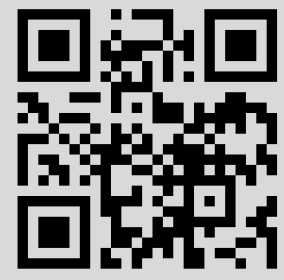




\section{О числе замкнутых геодезических на многограннике}

\section{В. Ю. Протасов}

Геодезической на поверхности в $\mathbb{R}^{3}$ называется спрямляемая жорданова кривая, локально-кратчайшая в каждой точке. Нас будут интересовать только замкнутые несамопересекающиеся геодезические, по умолчанию любую геодезическую будем считать таковой. В 1905 г. А. Пуанкаре выдвинул гипотезу о существовании трех геодезических на гладкой выпуклой поверхности. В 1930 г. гипотеза была доказана Л.А. Люстерником и Л.Г. Шнирельманом (см. [1]), позже было установлено существование бесконечного числа геодезических [2], [3]. Случай негладких поверхностей, в частности многогранников, исследовался в [4]-[10]. В отличие от гладких поверхностей, многогранник может вовсе не иметь геодезических [7]. Геодезические на многограннике суть ломаные с вершинами на ребрах, не проходящие через вершины многогранника и обладающие свойством отражения: любые два соседние звена ломаной образуют равные углы с соответствующим ребром. Далее вершины ломаной будем называть узлами. Если развернуть многогранник вдоль геодезической $g$ (последовательно разворачиваем грани на плоскость в том порядке, как их пересекает геодезическая), то $g$ перейдет в прямую на плоскости развертки. Две геодезические изоморфны, если они пересекают одну и ту же последовательность ребер. Если многогранник имеет одну геодезическую, то он имеет бесконечно много изоморфных ей геодезических; их длины равны, а соответствующие звенья параллельны. В дальнейшем будем отождествлять изоморфные геодезические и считать разные геодезические неизоморфными. Известно, что куб имеет три неизоморфные геодезические, а правильный октаэдр - две. Правильный симплекс имеет бесконечно много геодезических, все они классифицированы в [10]. То же верно и для равногранных симплексов (грани - равные треугольники). Неравногранный симплекс имеет конечное число геодезических, их число оценивается параметрами симплекса [10]. Возникает задача - описать все многогранники, имеющие бесконечно много геодезических (или, что то же, - сколь угодно длинные геодезические, или - геодезические со сколь угодно большим числом узлов).

Теорема 1. Многогранник имеет бесконечно много геодезических тогда и только тогда, когда он является равногранным симплексом.

ДокАзАТЕЛЬСтво. Пусть многогранник $G$ имеет геодезические со сколь угодно большим числом звеньев $N$. Произвольная геодезическая $g$ делит поверхность $G$ на

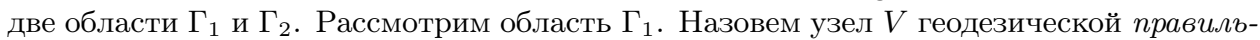
ным, если найдется узел $U$ такой, что интервал $V U$ лежит в $\Gamma_{1}$ и является частью некоторого ребра $G$. Каждое ребро может содержать не более двух неправильных узлов. Поэтому при $N \rightarrow \infty$ геодезическая $g$ будет иметь сколь угодно длинные участки, содержащие только правильные узлы $V_{1}, \ldots, V_{p}$. Для любого $i$ узлы $V_{i-1}$ и $V_{i}$ лежат на одной грани (на ребрах $c$ и $d$ ) и соединены звеном $a_{i}$. Назовем нарушением ситуацию, когда соответствующие узлы $U_{i-1}$ и $U_{i}$ не соединены звеном. Это значит, что из них выходят два разных звена, лежащие на данной грани по одну сторону от $a_{i}$. Поскольку $g$ не имеет самопересечений, каждой паре ребер $c$ и $d$ данной грани соответствует не более двух нарушений для $g$. Поэтому число всех нарушений ограничено. Следовательно, при $N \rightarrow \infty$ найдутся участки $g$ сколь угодно большой длины $L$, содержащие только правильные узлы $V_{1}, \ldots, V_{m-1}$ без нарушений, т. е. узлы $U_{1}, \ldots, U_{m-1}$ также будут последовательными узлами ломаной $g$. Развернув грани $G$

Работа выполнена при поддержке гранта РФФИ 08-01-00208, грантов МД-2195.2008.1 и НШ-3233.2008.1. 
на плоскость последовательно относительно ребер, содержащих данные узлы, получаем два отрезка: первый состоит из звеньев $a_{1}, \ldots, a_{m}$, второй - из звеньев $b_{1}, \ldots, b_{m}$, где $a_{i}$ - отрезок $V_{i-1} V_{i}$, а $b_{i}$ - отрезок $U_{i-1} U_{i}$. Можно считать, что выбранный участок правильных узлов - максимальный, т. е. узлы $V_{0}$ и $V_{m}$ - неправильные. В противном случае оба отрезка продолжаются неограниченно вдоль развертки, следовательно, $g$ не будет связна. При каждом $i$ звенья $a_{i}$ и $b_{i}$ лежат на одной грани, полоса между ними является частью $\Gamma_{1}$ и, следовательно, не содержит других точек $g$. Площадь полосы на плоскости развертки между отрезками $V_{0} V_{m}$ и $U_{0} U_{m}$ не превосходит площади $\Gamma_{1}$. Поэтому, выбирая длину $L$ достаточно большой, считаем, что расстояние между отрезками (максимальная ширина полосы), а также угол между ними не превосходят заданного $\varepsilon>0$. Обозначим через $\gamma$ грань, в которой лежат звенья $a_{m}$ и $b_{m}$. Поскольку узел $V_{m}$ не правильный, он не лежит на одном ребре с узлом $U_{m}$. Значит, некоторые вершины грани $\gamma$ находятся между $a_{m}$ и $b_{m}$. Если $\varepsilon$ мало, то между $a_{m}$ и $b_{m}$ может лежать только одна вершина, назовем ее $A$. Считаем, что $\varepsilon<2 \pi-\sigma(A)$, где $\sigma(A)$ - сумма плоских углов при вершине $A$. Пусть $s_{1}, \ldots, s_{k}-$ лучи, идущие вдоль ребер, выходящих из $A, V_{m} \in s_{1}, U_{m} \in s_{k}$. Продолжим геодезическую $g$ по плоскостям граней, сходящихся в вершине $A$, дальше за точку $V_{m}$, переходя через лучи $s_{1}, s_{2}, \ldots$ по свойству отражения. Предположим, она пересечет лучи $s_{1}, \ldots, s_{r}$ и не пересечет $s_{r+1}$. Это означает, что она выйдет из некоторого узла $V_{m+r-1} \in s_{r}$ и пойдет вдоль луча $\alpha$, не пересекающего луч $s_{r+1}$. Продолжим так же второй участок $g$ за точку $U_{m}$, переходя через лучи $s_{k}, s_{k-1}, \ldots$ Элементарным подсчетом углов, пользуясь неравенством $\varepsilon<2 \pi-\sigma(A)$, показываем, что это продолжение пересечет луч $s_{r+1}$ в некотором узле $U_{m+k-r-1}$, выйдет из этого узла вдоль некоторого луча $\beta$, пересекающего либо луч $s_{r}$ (в узле $U_{m+k-r}$ ), либо луч $\alpha$ (в некоторой точке $P$ ). Если $\varepsilon$ достаточно мало, то все новые узлы и точка $P$ лежат внутри соответствующих граней $G$. Если $\beta$ пересекает $\alpha$, то $g$ будет самопересекающейся; если $\beta$ пересекает $s_{r}$ и при этом $A U_{m+k-r}<A V_{m+r-1}$, то узел $U_{m+k-r}$ лежит внутри $\Gamma_{1}$, что также невозможно. Аналогично - со случаем $A U_{m+k-r}>A V_{m+r-1}$. Следовательно, узлы $U_{m+k-r}$ и $V_{m+r-1}$ совпадают и $g$ зацепляется за вершину $A$. Это означает, что $g$ пересекает все ребра, выходящие из $A$, причем точки пересечения являются последовательными узлами геодезической, и каждый из этих узлов является ближайшим к $A$ на соответствующем ребре. Тогда угол между отрезками $V_{0} V_{m}$ и $U_{0} U_{m}$ равен $|\sigma(A)-\pi|$. Так как этот угол меньше произвольного $\varepsilon$, то $\sigma(A)=\pi$. Значит, $V_{0} V_{m}$ и $U_{0} U_{m}$ параллельны. Проведя те же рассуждения с другого конца - с узлами $V_{0}$ и $U_{0}$, получаем, что $g$ зацепляется за некоторую другую вершину $B$. Итак, область $\Gamma_{1}$ содержит всего две вершины $A$ и $B$, причем $\sigma(A)=\sigma(B)=\pi$. Аналогично, $\Gamma_{2}$ содержит две вершины. Таким образом, многогранник $G$ имеет четыре вершины, т. е. это - симплекс, а так как сумма плоских углов при каждой вершине равна $\pi$, то этот симплекс равногранный.

\section{Список литературы}

[1] Л. А. Люстерник, Л. Г. Шнирельман, УМН, 2:1 (1947), 166-217. [2] J. Franks, Invent. Math., 108:2 (1992), 403-418. [3] V. Bangert, Internat. J. Math., 4:1 (1993), 1-10. [4] А. Д. Александров, Внутрення геометрия выпуклых поверхностей, ОГИЗ, М.-Л., 1948. [5] А. В. Погорелов, Матем. сб., 25:2 (1949), 275-306. [6] W. Klingenberg, Lectures on closed geodesics, Grundlehren Math. Wiss., 230, Springer-Verlag, Berlin-New York, 1978. [7] G. Galperin, Regul. Chaotic Dyn., 8:1 (2003), 45-58. [8] A. O. Ivanov, A. A. Tuzhilin, Acta Appl. Math., 66:3 (2001), 251-317. [9] A. V. Zorich, International Congress of Mathematicians, Vol. 3 (Madrid, 2006), Eur. Math. Soc., Zürich, 2006, 121-146. [10] В. Ю. Протасов, Матем. сб., 198:2 (2007), 103-120.

В. Ю. Протасов (V. Yu. Protasov)

Московский государственный университет им. М. В. Ломоносова

E-mail: v-protassov@yandex.ru
Представлено В. М. Тихомировым Принято редколлегией 20.06.2008 